\title{
Use of an Interdisciplinary Teaching to Innovation Model for an Agricultural Marketing
}

\author{
Hui-Jiun $\mathrm{Hu}$ and Li-Shu Lu
}

\begin{abstract}
This course through the collaboration of interdisciplinary teachers and experts. Professional techniques from various fields of knowledge were combined to stimulate innovative thinking among the students. The teaching method involved the "double diamond" design process model: Students explored (discover, 1D) the needs of the people, environment, and other items in agricultural areas; defined (define, 2D) problems that could be solved; participated in brainstorming activities to develop (develop, 3D) problem-solving directions and tools; and implemented their ideas and presented (deliver, 4D) their projects. Furthermore, the students were taught to utilize the convenient information and communication technologies (ICTs) that were available during the development of their projects. Not only can it reduce development costs, but it can also speed up development. We found the high-quality agricultural products produced by small farmers were found to have lacked brand and marketing channels; thus, they were unable to attract young people willing into the countryside. Finally, we proposed an innovative model of agricultural marketing: "Customized QR Code Video Marketing linking Mobile Payment Technology". Through the integration of PayPal's mobile payment technology with the customized QR code design and the marketing video upload system. Small farmers can able to easily engage in direct sales, improve the brand identity of their high-quality nontoxic products, and more flexibility reduces cost.
\end{abstract}

Index Terms-Interdisciplinary teaching, 4D teaching method, agricultural marketing.

\section{INTRODUCTION}

In 2010, the Rural Regeneration Act was promulgated in Taiwan to promote rural development, activation, and regeneration; improve basic production conditions; protect the rural ecology and culture; and enhance the quality of life in rural areas [1]. However, a major problem in the promotion of rural regeneration in Taiwan is the perception that rural areas do not hold any benefits, resulting in unwillingness among young people to enter the industry and a lack of channels to do so[2]. Thus, education is the most direct way to familiarize students with the countryside and rural areas and help them understand how they can contribute to these areas. Moreover, rural residents may also benefit from the students' creativity and talents. As such, a friendly bridge between young people and the countryside can be developed.

Furthermore, Przeclawski (1993) emphasized that dialogue

Manuscript received April 15, 2018; revised August 1, 2018.

Hui-Jiun Hu is with the Department of Visual Arts, National Chiayi University, Chiayi, Taiwan (e-mail: momo@mail.ncyu.edu.tw).

$\mathrm{Lu} \mathrm{Li}-\mathrm{Shu}$ is with the Department and Graduate School of Digital Media Design, National Yunlin University of Science and Technology, Douliou, Yunlin City, Taiwan (e-mail: luls@gemail.yuntech.edu.tw). between various academic disciplines can provide more diversity and opportunities [3]. An interdisciplinary collaboration teaching model enhanced students' professional skills in various fields of knowledge and enriched their theoretical and practical experience. Field knowledge, creative-thinking training, and smart technologies were incorporated in the course to cultivate exploratory skills in the students and teach them to utilize existing technologies. Opportunities for the creative design were introduced to develop a problem-solving innovative model.

\section{LITERATURE REVIEW}

\section{A. Interdisciplinary Knowledge}

With rapid technological advances and the diversification of society, a single-knowledge system is insufficient for addressing crucial domestic and global issues. A spark of innovation can be easily generated through the combination of perspectives and professions, including the use of various subjects; research areas; community members; and industry, government, and university representatives. Przeclawski (1993) suggested that cross-disciplinary research focuses on special issues and explores problems through collaboration between multiple academic fields. In cross-disciplinary research, scholars from varying fields form a research group They define the problem and provide the theoretical background, drawing on their individual professional expertise. This requires deep involvement, commitment, and consensus among the researchers in the research project [3]. Lee and Chang (2011) also claimed that the process of turning an idea into a product is challenging. In the past, it was not easy to fulfil market demand by relying on a single technology in industry development. The innovation of a technological product was achieved by combining technologies from multiple fields. Therefore, the integration of cross-disciplinary knowledge has become a key factor in ensuring competitiveness in research and development [4].

In addition, the course is taught through theoretical construction and teamwork. And practice the spirit of learning by doing interdisciplinary learning. The course leads students into the field and gives them the opportunity to gain an in-depth understanding of the community where the university has been studying and living for four years. Students learn about people, culture, land, scenery and production in this community. Further, they can care for the community, identify with the community, and foster a social entrepreneurial spirit of social care and enthusiasm. This study follows the 5C overall goal of the Smart Agricultural Extension Integration and Innovation Union (2016). The 
curriculum, combined with the community's field strength, through the communication and collaboration, and finally developed creative Design [5]. As shown in Fig. 1.

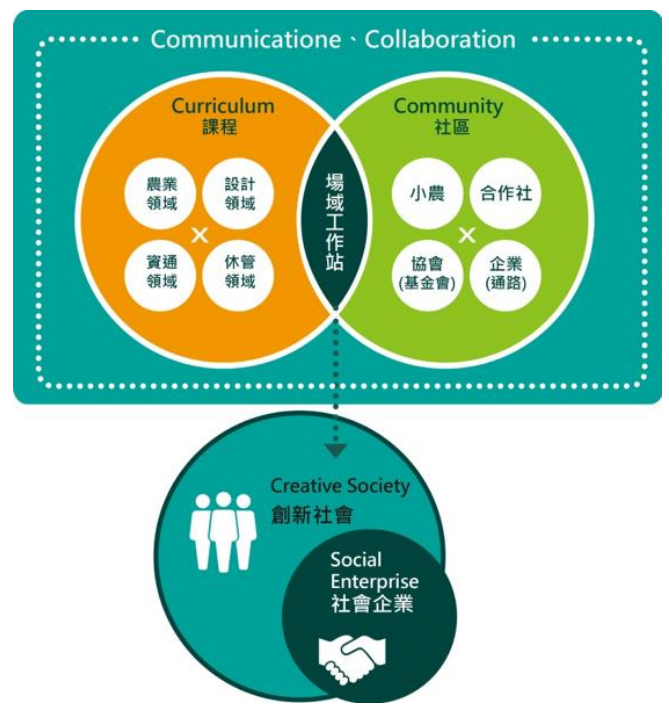

Fig. 1. Overall goal concept map of Smart Agricultural program- $\ulcorner 5 \mathrm{C} \rightarrow$ Social Enterprise $\lrcorner($ Union, S. A. E. I. a. I., 2016.) [5].

\section{B. Consumption Pattern in Change}

Novel modern service technologies can change the form of consumption. For instance, South Korean international supermarket chain Homeplus conducts research on local users' lifestyles and consumption behaviors. Advertisements for an array of products, including drinks, meat products, and fresh fruit, are placed in subway stations, accompanied by price information and QR codes. People waiting for a train in the metro station can browse a virtual store and scan the QR code of a favorite product using their phones. They can even purchase the product and have it delivered to their home (Fig. 2) [6]. This novel service model has attracted many new members and online customers to Homeplus, increasing its popularity and encouraging the purchase of goods on Homeplus.

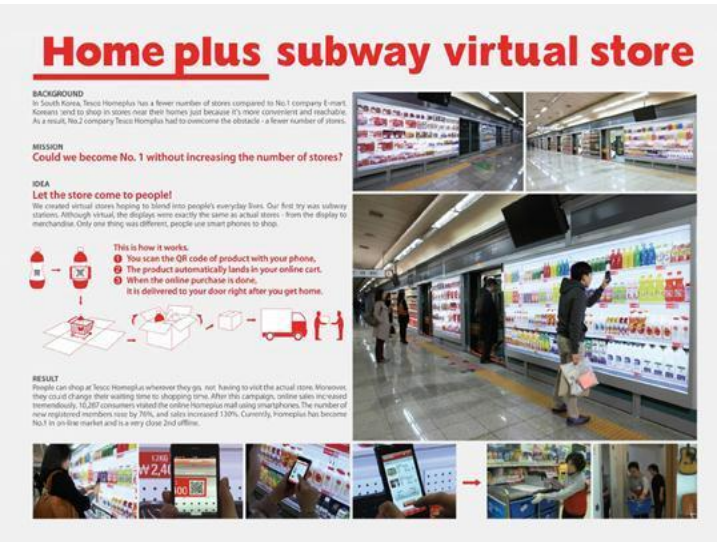

Fig. 2. HomePlus in South Korea uses QR codes and successfully establishes a virtual store in a subway [6].

In 2016, in China, Alibaba Group's payment system launched a Double 12 promotion that focused on physical retail stores. Customers only needed to make a transaction via Alipay to receive a $50 \%$ discount. This sparked an Online to Offline (O2O) mobile payments war [7]. Businesses no longer focus on price wars as they have switched their attention to service and creativity.

Smartphones have changed people's lifestyle habits. Handheld devices have provided a great degree of convenience and have changed business operation models and even cash flow models [8]. According to estimates reported in a study by Gartner, the scale of mobile payment transactions worldwide reached US $\$ 431.1$ billion in 2015 and $\$ 721.5$ billion in 2017 [9]. Mobile payments have provided the market with ample business opportunities and caused a reform in cash flow. Studies on related topics must examine consumption tendencies and technology application perspectives.

\section{TEACHING RESEARCH DESIGN}

\section{A. Course Design}

The participants of this course were 15 fourth-year university students who took the "Design Workshop" taught by Dr. Hu of the Department of Visual Arts. Professional techniques from various fields of knowledge were combined to stimulate innovative thinking among the students. The 18-week course plan includes field visits, in-depth interviews with small farmers, expert lectures, SEM strategy experience thinking, creative design methods, learning , and application of information communication technology, concept design proposals, and design practices, etc. (Fig. 3).

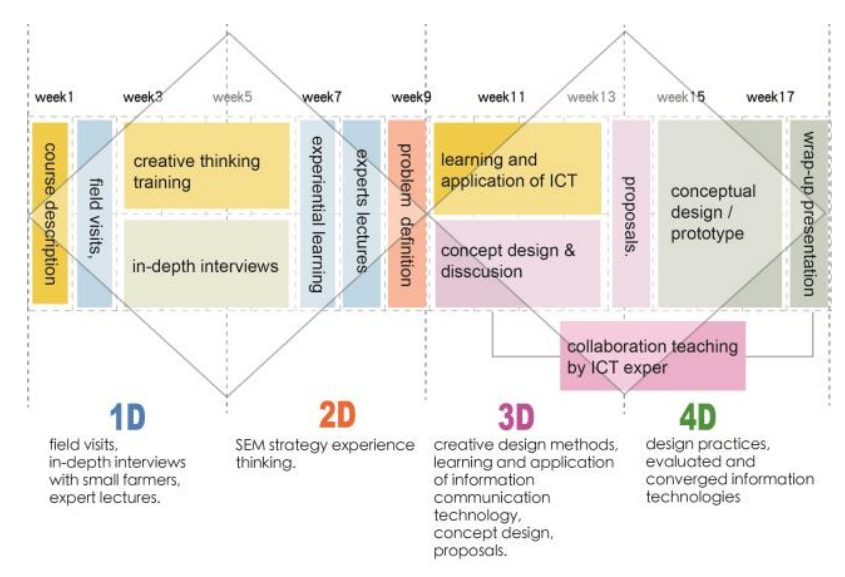

Fig. 3. The framework of 18-week course (data source: this study).

In this course, I invited the director-general of Shanglin Community to give a guided tour. Mr. Lin, who is a non-toxic farmer, made a farm introduction, and Prof. $\mathrm{Hu}$, who curated the Yunlin Agricultural Expo, gave an expert lecture. Then, we visited the Wujia Bee Farm, Zhongpu Community, and Minxiong Community. And, I invited Prof. Chang from the Department of Information Management conducts ICT teaching and implementation in my class. The course design is based on the collaborative of interdisciplinary teachers and experts, bringing design thinking that is "user-centered". From problem-finding, problem-solving, creative thinking, aesthetic design, and smart technologies were integrated. It guides young people to propose innovative designs that are broader that corresponded with current demand. It can implement the concept of local practice, and integration form creative aesthetics and wisdom technology. 


\section{B. 4D Teaching Method}

The teaching method involved the Design Council's "double diamond" design process model (4Ds): Students explored (Discover, 1D) the needs of the people, environment, and other items in agricultural areas; defined (Define, 2D) problems that could be solved; participated in brainstorming activities to develop (Develop, 3D) problem-solving directions and tools; and implemented their ideas and presented (Deliver, 4D) their projects [10]. The double diamond design considers "double divergence-convergence" in the teaching process. The first diamond involves the divergence and convergence processes (about what and why) related to finding correctly defined problems; whereas the second diamond involves the divergence and convergence processes (about how) related to the development of practical solutions to people's needs. The introduction of these two vital design steps helps to generate more diverse ideas. The 4D teaching content of this course is planned as shown in Fig. 4. 1D (discover) includes field visits, experiential learning, interviews, experts lectures. 2D (define) includes comprehensive analysis, communication convergence, problem definition. 3D (develop) includes creative thinking, ICT teaching, teamwork. 4D (deliver) includes collaboration creation, technology integration, innovation practice.

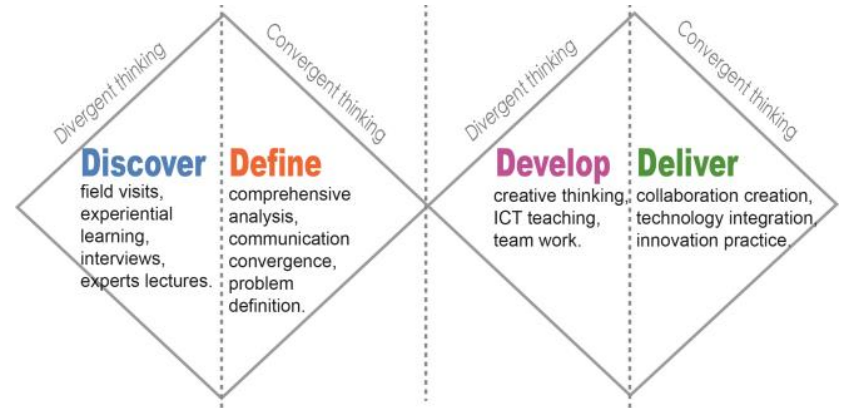

Fig. 4. 4D teaching design of the course (data source: this study).

\section{Teaching Process}

\section{(1) Step 1: Discover}

The main task in design is understanding the problem [11]. According to Brown (2009), the first step in design is to directly observe and understand the work, living, and community environments of consumers, users, and customers to uncover the needs that they may be unable to describe. The design must be person-centric and must consider people's behaviors, needs, and preferences, as well as the feasibility of the technology and business. Such comprehensive thinking is necessary to match innovative concepts to people's needs and ideas and, thus, create value [12]. Therefore, community visits, interactive agricultural experiences, and interviews with farmers were arranged during the course to fully familiarize students with the local people, culture, landscape, scenery, and industry. A large amount of data was collected through observations and interviews. Practical experience, on-site visits, and conversations with small farmers provided a better understanding of the farmers' production models and the special characteristics of agriculture, which helped the students to identify the true problems, needs, and aspirations of people living in rural areas. Fig. 5 shows the director-general of Shanglin Community to give a guided tour.
Fig. 6 shows Mr. Lin, who is a non-toxic farmer, made a farm introduction. Fig. 7 shows we visited the Wujia Bee Farm, Zhongpu Community, and Minxiong Community. Fig. 8 shows Prof. Hu, who curated the Yunlin Agricultural Expo, gave an expert lecture.

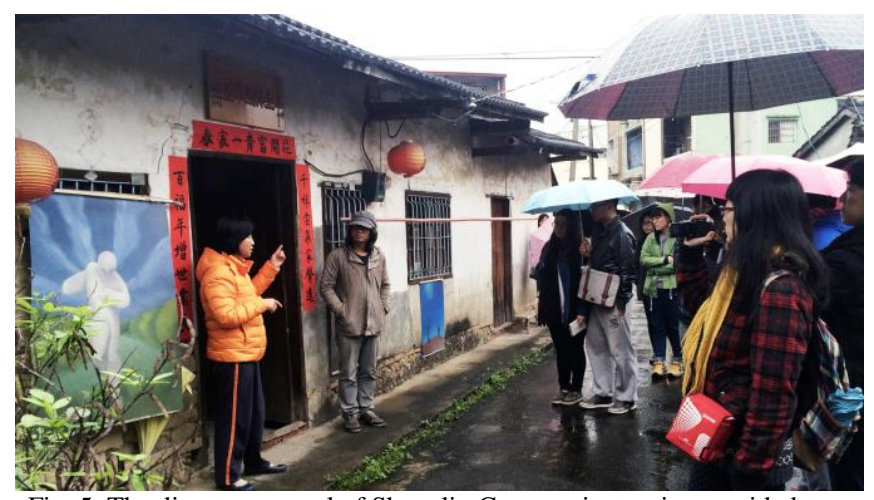

Fig. 5. The director-general of Shanglin Community to give a guided tour (data source: this study).

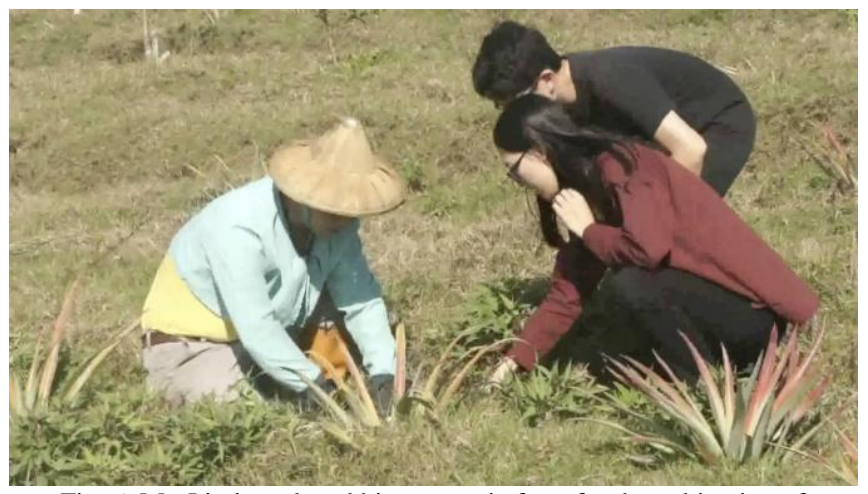

Fig. 6. Mr. Lin introduced his non-toxic farm for the cultivation of pineapples in grasslands (data source: this study).

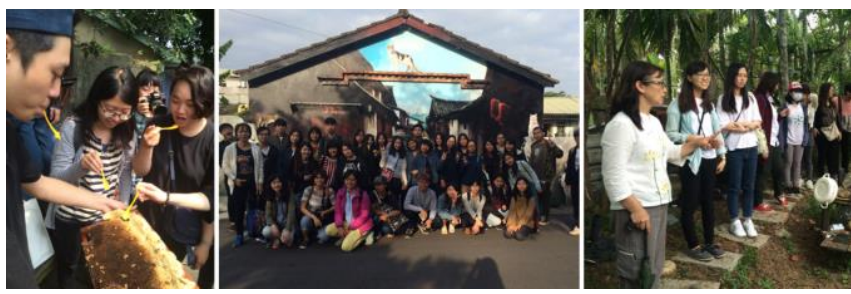

Fig. 7. visited the Wujia Bee Farm (left), Zhongpu Community (middle), and Minxiong Community (right) (data source: this study).

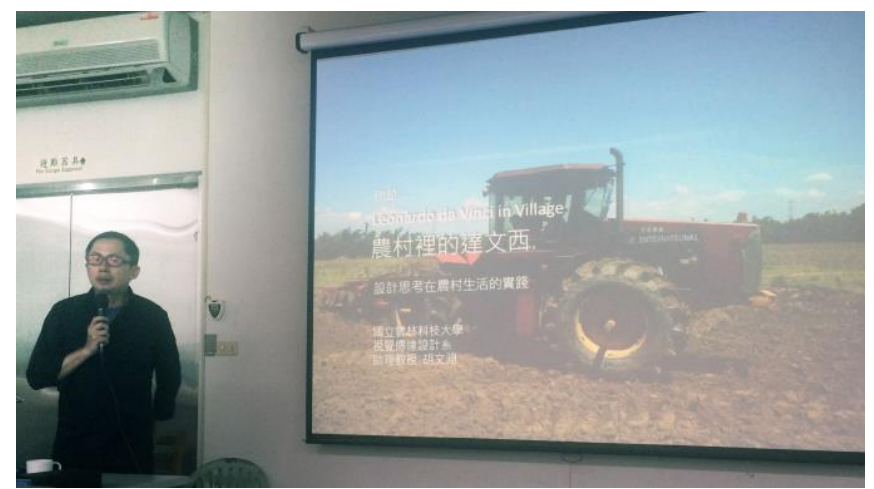

Fig. 8. Prof. Hu's expert lecture the Yunlin Agricultural Expo (data source: this study).

(2) Step 2: Define

The students were taught to determine which possibility discovered during the "Discover" step was the most crucial, what action needed to be taken first, and which problems could be solved? Furthermore, the course also taught 
Strategic Experiential Modules (SEMs) proposed by Schmitt (1999); that is, group thinking was implemented using five aspects: sense, feel, think, act, and relate. He indicated a product can compete on the market only if it can provide consumers with experiences they truly desire, for this will not only appeal to their feelings but also increase their willingness to purchase [13].

Then, students discussed and clarified problems with small farmers many times (Fig. 9). We returned to the classroom with more information for discussion, and analysis. We used SEMs to summarized and defined the problems based on local culture, history, character, industry, strengths, weaknesses, opportunities, threats, and symbolic elements (Fig. 10). Finally, we define the topics to be solved in this course: how to improve the brand identity of small farmers and the marketing model of agricultural.

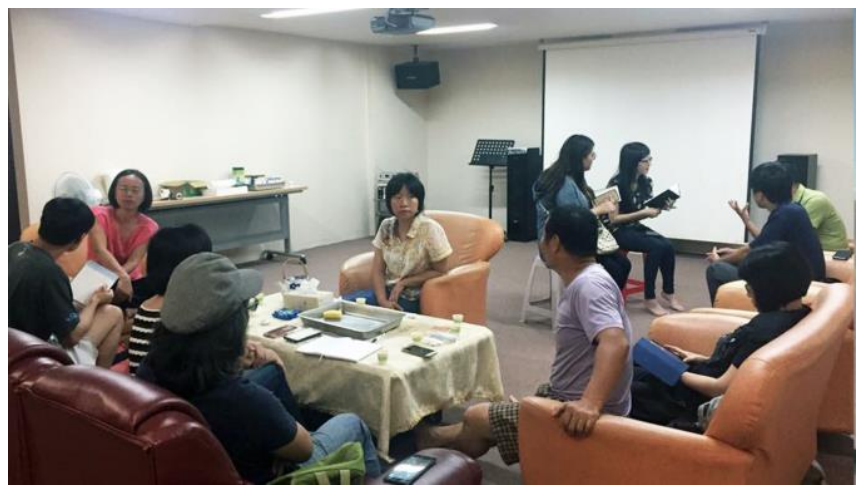

Fig. 9. Students discussed and clarified problems with small farmers (data source: this study).

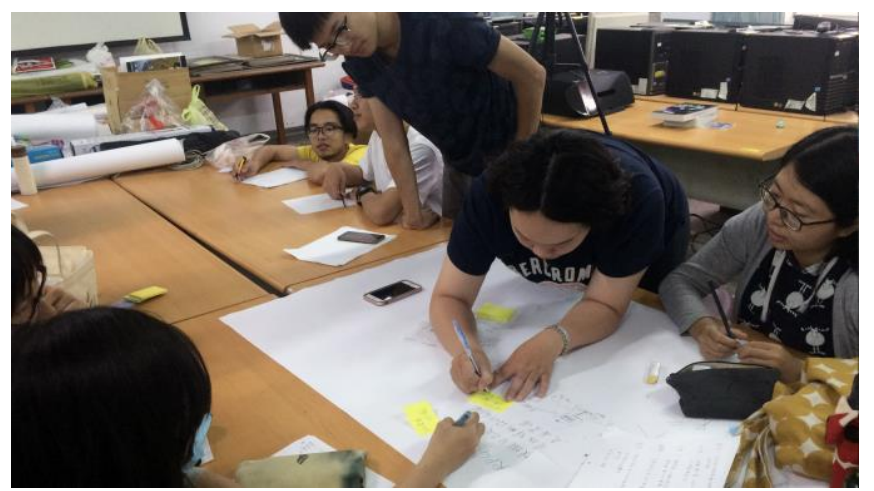

Fig. 10. Used SEMs to summarized and defined (data source: this study).

(3) Step 3: Develop

This stage emphasizes how to solve problems. First, divide the class students into two teams for design development. Then, we are through a large number of vertical and horizontal brainstorming activities (such as observation, bionic design, association method, mind mapping and other creative thinking methods) (Fig. 11), and collaboration teaching by information technology experts (Fig. 12). Prof. Chang from the Department of Management Information Systems was invited to collaboratively teach (four weeks of collaborative classes and two weeks of joint instruction). Students have the opportunity to learn about the Internet of Things (IoT) technologies, interactive design technologies, and Quick Response Code (QR code) applications etc. Furthermore, the students were taught to utilize the convenient information and communication technologies (ICTs) that were available during the development of their projects. Finally, the students proposed many innovative ideas of possibilities for design and technology integration.

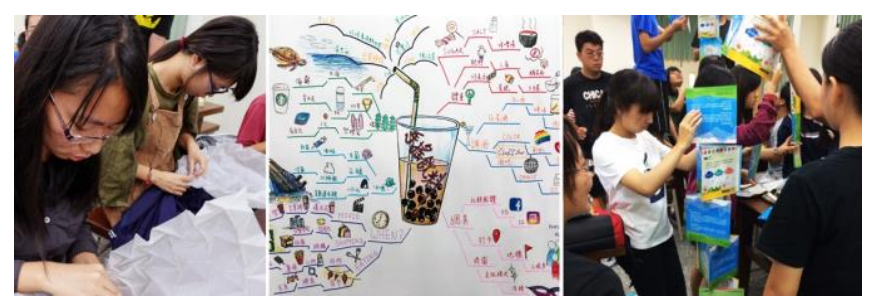

Fig. 11. creative thinking methods. bionic design (left), mind mapping (middle), teamwork competition (right). (data source: this study).

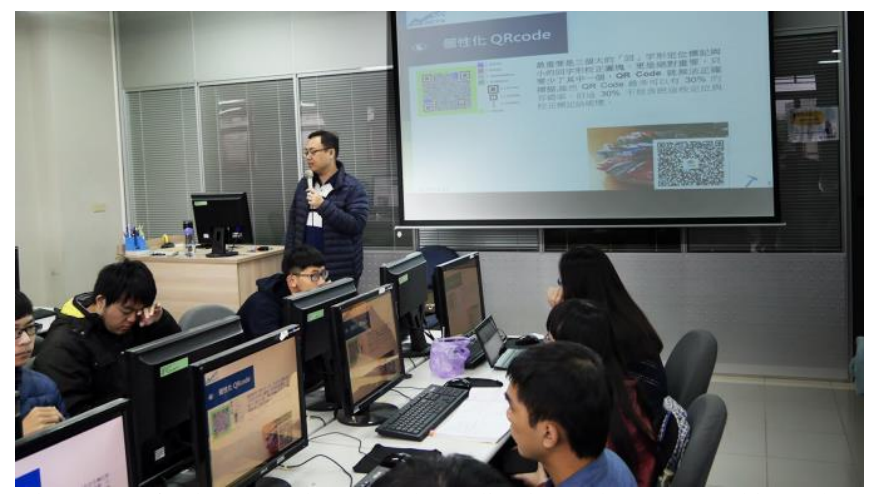

Fig. 12. Prof. Chang taught QR code applications (data source: this study).

\section{(4) Step 4: Deliver}

The various interesting innovative ideas proposed during the "Develop" step was evaluated and converged using information technologies. With the support of teamwork and multi-teachers' guidance, a final innovative design model was developed, which combined visual aesthetics and smart technologies (Fig. 13).

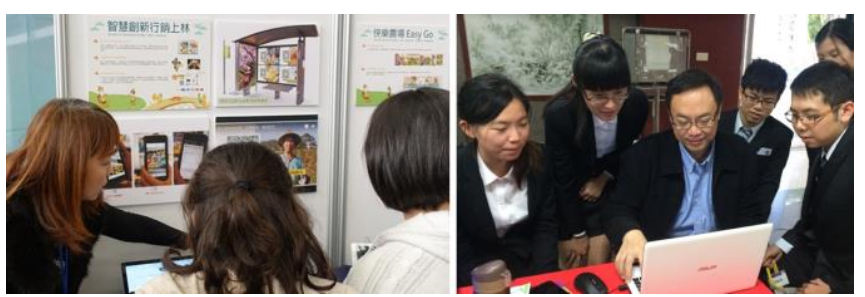

Fig. 13. Teachers collaboratively guidance for teamwork. Visual Art teacher (left), ICT teacher (right) (data source: this study).

\section{THE PRACTICE OF INNOVATIVE MODEL FOR AN AGRICULTURAL MARKETING}

The Shanglin community, which was the partnering community for this study, resides in Nanbian Village, Dalin Township, Chiayi County, Taiwan. The village consists mostly of low-rise buildings made from red bricks and retains many traditional bamboo-framed buildings (constructed using traditional techniques) that give the village a simple rural style. The 6-hectare pond in the area serves not only as the water source for crops, but also as the home of a rich ecological community and a scenic spot suitable for watching sunsets. Most of the residents here are farmers whose main crops include rice, bamboo shoots, and pineapples. In recent years, the cultivation of the cape jasmine for use as a traditional natural dye was started, and active efforts have been made to turn it into a local specialty product. 2016 saw Dalin Township earning the Cittaslow International certification, making the township the first "slow living town" in western 
Taiwan. However, the substantial outflow of young adults and the rapidly aging local population (adults aged 65 or older make up $26 \%$ of the population) mean that this community is a typical aging community.

According to this course discovery were as follows: Despite the small farmers' growing focus on ecology and shift to organic farming, high-quality agricultural products lacked effective marketing channels and were sold to wholesalers, which led to a reduction in profits, an absence of branding, and an inability to attract young people to venture into the countryside. Finally, through the collaboration of interdisciplinary teachers and experts, induction of the creative proposals of the teamwork. The following three solutions were proposed in response to this problem.

\section{A. Solution 1: Design of Customized QR Codes for Small Farmers}

The design and use of customized QR codes can help establish a personal identity and image for agricultural products produced by small farmers. The QR code is the two-dimensional barcode invented by Japanese company Denso-Wave in 1994 [14]. QR codes have a square shape, with data recorded in both their length and width; QR codes are usually black-and-white images. The maximum false acceptance rate (FAR) of a QR code is $30 \%$. Homocentric squares appearing in the corners are position symbols, and smaller homocentric squares are calibration blocks. If a calibration block is missing, the QR code cannot be scanned correctly. Fig. 14 shows the basic information provided by a QR code. In this study, a QR code's FAR was used to design a special logo for small farmers, comprising less than $30 \%$ of the QR code content. As such, a customized QR code for small farmers was created (Fig. 15).

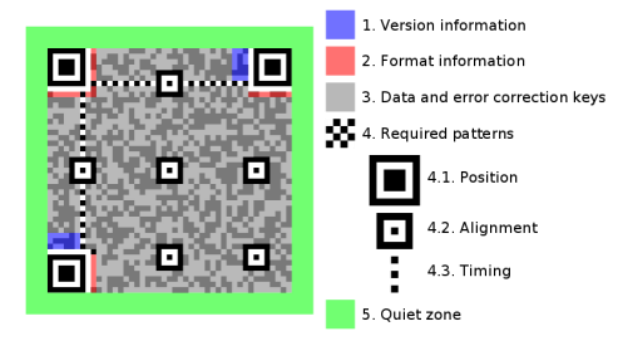

Fig. 14. Structure of a QR code, highlighting functional elements [14].

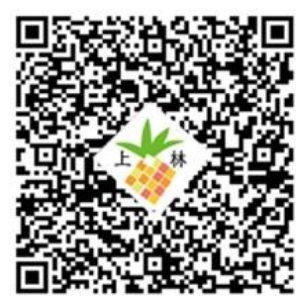

Fig. 15. Customized QR code for small farmers (data source: this study).

\section{B. Solution 2: Creation of Marketing Videos for Agricultural Product Stories}

With the rapid development of the Internet and smart communication devices in recent years, watching videos on computers, tablets, and smartphones has become a new trend, giving rise to new types of media propaganda. Small farmers must consider new ways of presenting their stories. As stated by Jauyi Wu in CommonWealth Magazine, stories aim to sell and, thus, cannot be fictitious; stories make an impression when they feel real [15]. In this study, a marketing video, "Gold Hidden in Grasslands," for nontoxic garden-grown pineapples produced by Mr. Lin was created. The video presented a story on the local culture in which Lin was raised and described the unique techniques used for the cultivation of pineapples in grasslands, demonstrating Lin's care for the land, which increased the originality and distinctiveness of the product. A video script was developed based on the story (Fig. 16). After a discussion between the director, actors, and cameramen, the video shoot began on-site (Fig. 10). Finally, video post-production software was used to create the montage, subtitles, special effects, and on the video add customized QR code of small farmers. The completed video was uploaded to YouTube (Fig. 17) (URL: https://youtu.be/xBBO30W_hkY).

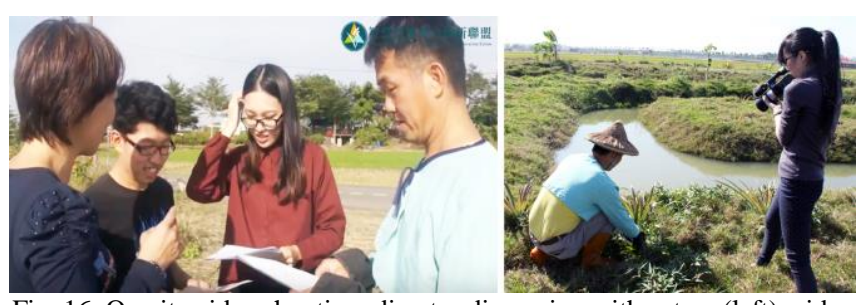

Fig. 16. On-site video shooting. director discussion with actors (left), video shoot (right) ) (data source: this study).

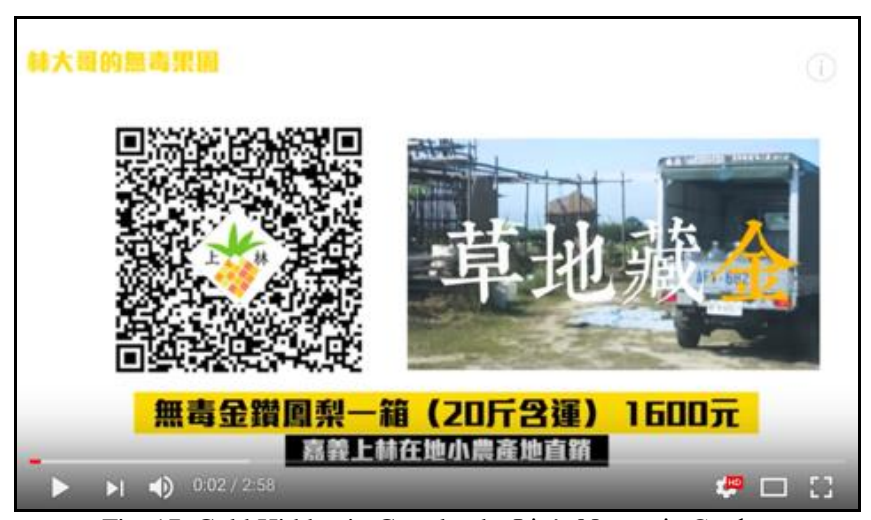

Fig. 17. Gold Hidden in Grasslands: Lin's Nontoxic Garden (data source: this study).

\section{Solution 3: Establishment of a Mobile Payment Platform}

In the next step, the agricultural product was uploaded to a marketing platform. To suit small farmers' businesses, the authors of this paper carefully evaluated the time costs, the integrity of the logistics and cash flow, the convenience of data maintenance, and universality of marketing initiatives. It was then decided that PayPal would be utilized. PayPal is a fast and safe cross-national third-party payment gateway used for online purchases and sales. To date, PayPal has over 200 million users and supports transactions in over 20 currencies. Convenient online cash flow does not require monthly fees [16]. PayPal's clear interface and simple uploading system made it a suitable choice for this course. An account can be registered according to the user's needs (Fig. 18), followed by the generation of a mobile payment QR code for the product in question (Fig. 19). Subsequently, the product is linked to a marketing video, which is then uploaded (Fig. 20). Finally, consumers may purchase the product via three simple steps: 
open, scan, and pay (open a QR code scanning program, scan the product's QR code to enter the product's sale page, and pay using a credit card) (Fig. 21).

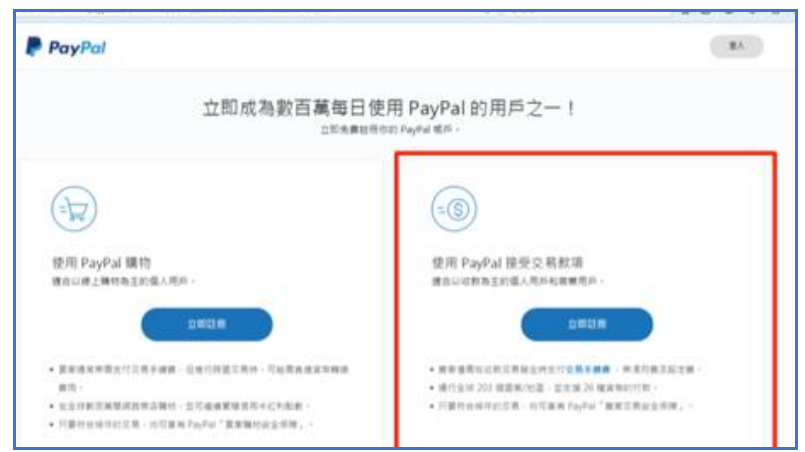

Fig. 18. PayPal registration (data source: this study).

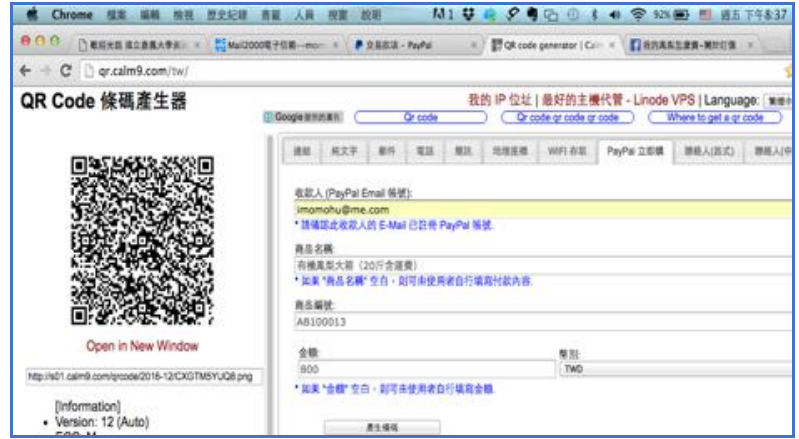

Fig. 19. Generation of a mobile payment QR code (data source: this study).

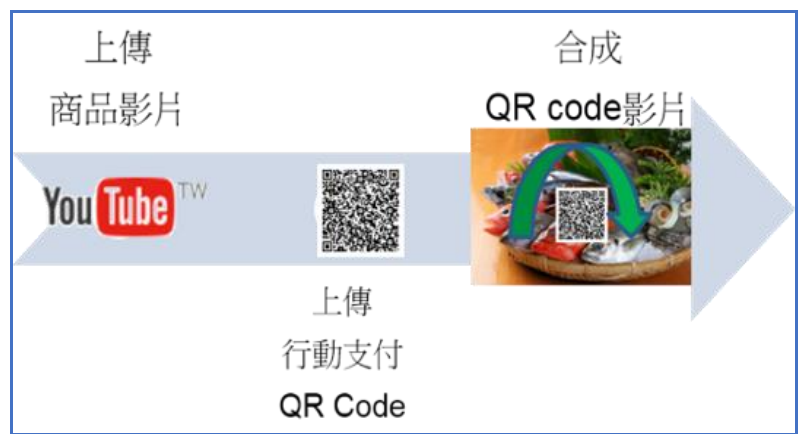

Fig. 20. Uploading the marketing video with the QR code (data source: this study).
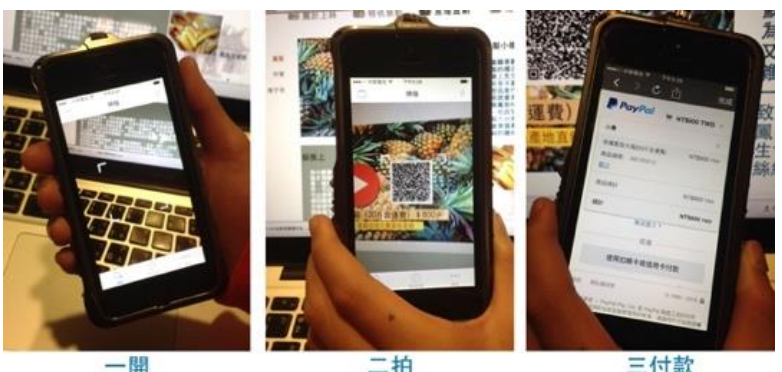

Fig. 21. Open, scan, and pay on a phone (data source: this study).

\section{Summary}

The design of a customized QR code improved brand identity. The production and uploading of a special agricultural marketing video and the integration of PayPal for mobile payments made it easy for the small farmers to engage in direct sales and improved the brand identity of their high-quality nontoxic products (Fig. 22). In addition to facilitating the purchase of high-quality agricultural produce by consumers, the project resolved the problems related to the agricultural products' marketing and sales channels. In the modern era of online and offline mobile payments, the concept of customized QR code visual marketing can be used to increase sales in shopping centers. In future, public transport stations, and other spaces with a large flow of people (Fig. 23), The marketing model will create greater value for small farmers and provide consumers with a more convenient service.

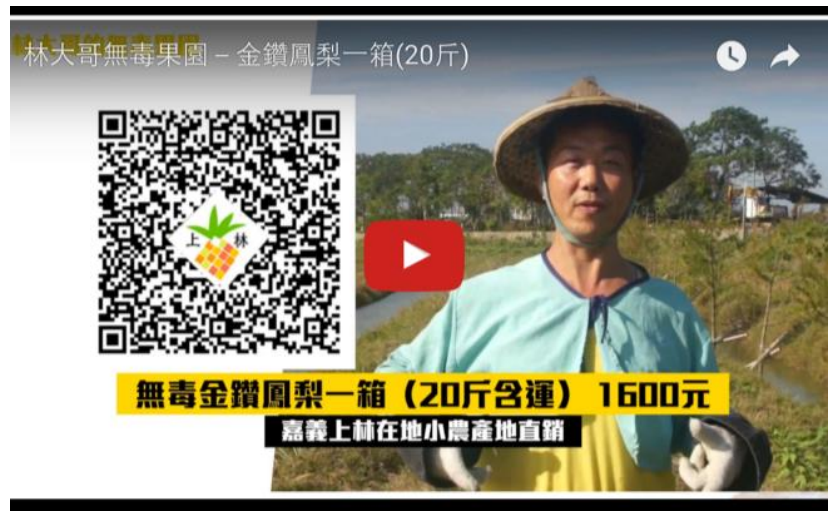

Fig. 22. Customized QR code video marketing (data source: this study).

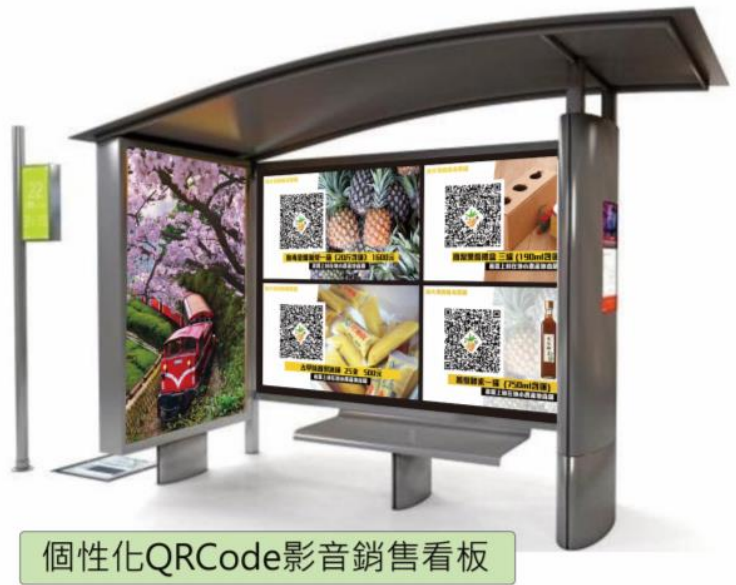

Fig. 23. The video marketing board of customized QR code (data source: this study).

\section{CONCLUSION AND SUGGESTIONS}

This course integrated various knowledge systems to cultivate practical skills in students and guide them toward becoming socially conscious and enthusiastic with innovative development skills. The students were taught to be smart creators rather than just knowledgeable workers.

Through this course, students explored the marketing problems faced by small farmers in the Shanglin community. The high-quality agricultural products produced by small farmers were found to have lacked effective exposure and marketing channels; thus, they were unable to attract young people to venture into the countryside. Creativity and smart technologies were integrated and an innovative agricultural marketing service model, "Customized QR Code Video Marketing \& Mobile Payment Technology,” was proposed. Through the integration of PayPal's mobile payment technology with the customized QR code design and the marketing video upload system, the small farmers were able to easily engage in direct sales, improve the brand identity of their high-quality nontoxic products, and reduce channel costs 
and supplier exploitation. Going forward, $\mathrm{O} 2 \mathrm{O}$ comprehensive marketing can be achieved by linking the products to physical stores and advertisement boards in MRT and bus stations.

In addition to the proposal of an innovative agricultural marketing service model to address small farmers' problems, the following benefits were achieved via the course: (1) interdisciplinary teaching methods can broaden the students' innovation knowledge and skills; (2) incorporation of the 4Ds design process into the course, it can provide the students with comprehensive design skills related to discovery, definition, development, and delivery; (3) interaction with farmers can cultivate active, enthusiastic, and socially conscious individuals with practical innovation skills. And, let the farmers perceive the value of university students. Furthermore, the students realized that regardless of whether they studied, worked in, or ventured into the countryside in the future, the resources of this rural society would always be waiting for them.

\section{ACKNOWLEDGMENT}

We would like to thank the Ministry of Education for the funding support that it has granted via the 2018 Talent Cultivation Program for the Smart Living Industry, the Smart Agricultural Extension Integration and Innovation Union for the courses that its core and partner schools have provided, and Prof. Chang Hong-Yi for the technical guidance that he has provided with respect to cooperative teaching.

\section{REFERENCES}

[1] E. Y. Council of Agriculture. (2010). Rural Regeneration Act. [Online]. Available: http://law.coa.gov.tw/GLRSnewsout/LawContent.aspx?id=GL000149

[2] W. Y. Ting, "Innovative agricultural marketing and the promotion of rural migration - A focus on people to attract youths to return," Agriculture Policy \& Review, vol. 297, 2017.

[3] K. Przeclawski, "Tourism as the subject of inter-disciplinary research, in tourism research," Tourism Research, London: Routledge, 1993, chapter 2 .

[4] R. H. Lee and Y. J. Chang, "Orange design and orange technology Innovation value-added and implementation," Science Development, no. 466, pp. 24-29, 2011/10.

[5] Union, S. A. E. I. A. I. 105 Smart Life Integration and Innovation Teaching Alliance Promotion Program, National Yunlin University of Science and Technology, 2016.
[6] Cheil. (2011). HomePlus Subway Virtual Store. [Online]. Available: https://www.adsoftheworld.com/media/outdoor/homeplus_subway_vi rtual_store

[7] Y. Y. Liao, "The battle rages on online and offline! China's 12-12 mobile payment war," 2016.

[8] F. W. Cheng, "Creating a new lifestyle with mobile payments. Online and offline payment applications," 2015.

[9] M. L. Chung, "The rise of mobile payment!" 2017.

[10] D. Council, Eleven Lessons-Managing Design in Eleven Global Brands: The Design Process, 2005.

[11] D. A. Norman, Emotional Design: Why We Love (or Hate ) Everyday Things, New York: Basic Books, 2004

[12] T. Brown, "Thinking like a designer," Commonwealth Magazine, 2009.

[13] B. H. Schmitt, Experiential Marketing: How to Get Customers to Sense, Feel, Think, Act, and Relate to Your Company and Brands, New York: The Free Press, 1999.

[14] Zephyris. (2013). Structure of a QR Code, Highlighting Functional Elements. [Online]. Available: https://commons.wikimedia.org/wiki/File:QR_Code_Structure_Exam ple_2.svg

[15] C. Y. Wu, "Telling the story of a product," Commonwealth Magazine, 2012.

[16] PayPal. (1999). A cross-border third-party payment platform that is safer and simpler. [Online]. Available: https://www.paypal.com/tw/webapps/mpp/merchant

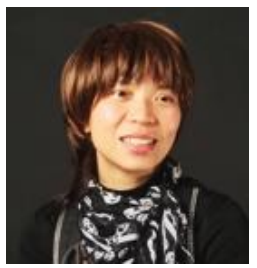

Hui-Jiun Hu is a Ph.D. in design from National Yunlin University of Science and Technology in Taiwan. And she is an associate professor with the Department of Visual Arts, National Chiayi University. Her research interests include visual communication design, user experience, UCD design, interactive design, design education, and technology art. In recent years, she has focused on teaching and research in interdisciplinary collaboration. Combine visual aesthetic design and technology to solve the inconvenient issues of the elderly, and community design.

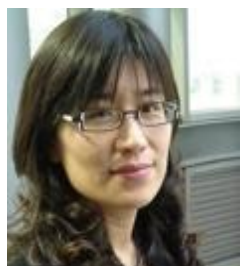

Li-Shu Lu is an associate professor of digital media Design Department and Graduate School of Computational Design at the National Yunlin University of Science \& Technology (YunTech) in Touliu, Taiwan, R.O.C. Miss Lu is also Chief of Information Technology Services Center. Her research interest is design education; design method $\&$ creative thinking; interactive Design; and usability in the interactive exhibition. In addition, in the context of the soundscape is also very interested. Miss Lu served as a designer in the design industry in July 1992 and June 1996, then, in 1997 transform in design education has been 20 years, and full of enthusiasm for teaching. Miss Lu has authored three books and authored, co-authored, or presented over forty-five articles, and conference presentations. 